\title{
Polanyi's tacit knowing and the relevance of epistemology to clinical medicine
}

\author{
Stephen G. Henry MD \\ Research Fellow, Department of Veterans Affairs Ann Arbor Healthcare System, University of Michigan Health System, Ann Arbor, Michigan, USA
}

\author{
Keywords \\ clinical judgment, epistemology, medical \\ knowledge, Michael Polanyi, philosophy of \\ medicine, tacit knowing \\ Correspondence \\ Stephen G. Henry \\ Department of Internal Medicine \\ University of Michigan \\ Robert Wood Johnson Foundation Clinical \\ Scholars Program \\ 6312 Medical Science Building 1 \\ 1150 W. Medical Center Drive \\ Ann Arbor \\ MI 48109-5604 \\ USA \\ E-mail: henrstep@med.umich.edu \\ Accepted for publication: 19 December \\ 2009 \\ doi:10.1111/j.1365-2753.2010.01387.x
}

\begin{abstract}
Most clinicians take for granted a simple, reductionist understanding of medical knowledge that is at odds with how they actually practice medicine; routine medical decisions incorporate more complicated kinds of information than most standard accounts of medical reasoning suggest. A better understanding of the structure and function of knowledge in medicine can lead to practical improvements in clinical medicine. This understanding requires some familiarity with epistemology, the study of knowledge and its structure, in medicine. Michael Polanyi's theory of tacit knowing is advanced as the basis for developing a more accurate understanding of medical knowledge. Tacit knowing, which explores the taken-for-granted background knowledge that underlies all human knowing, is explained in detail with a focus on its relevance for clinical medicine. The implications of recognizing tacit knowing in medicine and medical decisions are discussed. These include the ability to explain the importance of the clinical encounter in medical practice, mechanisms for analysing patient and doctor as persons, and the need for humility given the uncertainty that the tacit dimension injects into all medical decisions. This more robust medical epistemology allows clinicians to better articulate the nature and importance of patient-centred care, to avoid pitfalls inherent in reductionist approaches to medical knowledge, and to think more clearly about the relationships between medicine and health care at the individual and population levels.
\end{abstract}

During a typical day clinicians navigate dozens of medical problems that do not involve any grave illness or dramatic therapeutic dilemma. Routine cases, however, involve complex reasoning that clinicians tend to take for granted. Consider the following case:

A 38 year-old woman comes to your office complaining of drooling and inability to move the right side of her face.

Symptoms began abruptly two days ago after a family camping trip. She notes dry eyes and increased sensitivity to sound but denies pain or other medical problems.

This description suggests Bell's palsy, a fairly common problem in neurology and general practice that most clinicians, regardless of speciality, likely recall from medical school exams. Diagnosing Bell's palsy is neither unusual nor particularly difficult, so most clinicians would not bother to ask themselves how they know that a patient has Bell's palsy. Understanding how clinicians know what they know, however, is actually quite tricky. That simple question conceals many assumptions and controversies about knowledge and knowing that busy clinicians might dismiss as theoretical problems best left for philosophers and academics untroubled by the demands of everyday medical practice. Most clinicians tend to take for granted that routine medical practice is a 'real world', pragmatic, scientific undertaking whose conceptual underpinnings may be interesting but are generally trivial, selfevident, or irrelevant to decisions about patient care [1].

This perspective unfortunately leaves clinicians vulnerable to the influences of what MacIntyre called 'unrecognized theoretical ghosts' [2] that unknowingly shape their thoughts and actions. I shall argue that clinicians can exorcise one of these ghosts by investing a little time thinking about how and what they know, and about how their knowledge functions in everyday medical practice. Doctors learn and practice in an environment that proclaims the reductionist notion of medicine as applied science, despite a large body of literature demonstrating that medical practice has more in common with the social sciences than with the standard account of hypothesis-driven, value-free science [1,3,4]. This common misperception and the corollary that 'real' knowledge comprises discrete information that can be completely described through formal statements and explicit analysis creates a disconnect between how clinicians actually provide appropriate, patientcentred care in everyday practice and how they tend to think and write about medicine in the abstract. 
Repairing this disconnect requires a foray into epistemology, 'the study of the nature, sources and limits of knowledge' [5]. The abstract, reductionist view of medicine presupposes beliefs about knowledge that on close examination are inadequate to explain how clinicians handle even the simple case of a patient with Bell's palsy. Most clinicians' de facto understanding of medical epistemology cannot explain, for example, how understanding interactions among patients and clinicians is fundamentally different from solving complex mathematical or statistical problems. Clinicians whose understanding of medical knowledge accurately reflects clinical reality will be less vulnerable to the siren songs of reductionist epistemologies that lead many clinicians astray [1,6-8]. Michael Polanyi's concept of tacit knowing provides a starting point for constructing an epistemology of medical practice that is rigorous and accessible to clinicians without extensive philosophical training. A detailed analysis of how Polanyi's thought relates to medicine can explain and even predict many of the tensions between everyday practice and medical theory. Along the way, it also reinforces the traditional belief that face-to-face clinical encounters between patient and clinician are theoretically and substantively at the heart of medicine and suggests ways for promoting medical decisions that, by acknowledging the role of the person in medicine, are better tailored to individual patients' needs.

\section{Polanyi's theory of tacit knowing}

Michael Polanyi was a chemist and philosopher most well known for developing the concept of tacit knowing [9]. Tacit knowing refers to knowledge that functions at the periphery of attention and makes possible the conventionally recognized explicit domains of human knowledge. The clinician faced with the Bell's palsy patient described above uses many different kinds of information to understand her predicament. He concentrates directly on certain aspects of her case and notices others only indirectly or tacitly. The clinician listens closely to the story of her camping trip but notices individual words only to the extent that they make the story intelligible. During the neurological exam he sees her eyes, skin and teeth, but only insofar as they provide information about her facial nerve. He is unlikely to remember her eye colour even after a careful eye exam. These indirectly appreciated background details are part of what Polanyi called the tacit dimension of human knowledge. They form a largely taken-for-granted foundation that makes the information on which the clinician focuses directly, such as the patient's story and the significance of her neurological exam, possible. In other words, understanding the patient's story presupposes a tacit understanding of her spoken words and gestures; the clinician attends from the details of the patient's clinical presentation to the conclusion that she likely has Bell's palsy.

Two features of tacit knowing are especially relevant to clinical medicine. First, explicit knowledge cannot exist without a tacit background. One cannot make sense of a neurological exam without tacit awareness of the patient's body parts and how they are connected. A clinician who shifts his focus from the patient's neurological exam to her skin colour or clothing will tend to lose track of his exam findings. The exam's interpretation depends on certain kinds of details remaining in the background and tends to fall apart if those details become the centre of attention. The tacit dimension is a by-product of the directional structure of human interaction with the outside world (i.e. attending from background details to overall significance). The same information - skin colour - can function either tacitly or explicitly depending on how the clinician directs his focus, but some underlying tacit component is always present $[9,10]$.

Second, just how tacit particulars give rise to explicit knowledge cannot be fully captured in formal models or discrete steps; the relationship is ultimately inarticulable. As Polanyi wrote, 'we can know more than we can tell' [11] about the explicit conclusions reached from tacit awareness of a case. The clinician may note that the patient is upset and worried about her face, but he will be hard pressed to explain exactly how her voice, facial expressions and body language convey that impression. Even if he lists all the individual features he notices that communicate distress, many other signals that influence his judgments will remain unnoticed or overlooked. The tacit dimension by its nature tends to be taken for granted and so resists direct scrutiny or formal analysis. The tacit dimension is so pervasive that in everyday practice experienced clinicians often take for granted not only how recognition of particular facial features allows the successful diagnosis of Bell's palsy, but even that such a process takes place at all.

The tacit dimension has its roots in the human body. People walk, talk and eat with minimal appreciation for how their bodies accomplish these tasks; they do not coordinate individual muscle movements to chew gum or think about their vocal cords when calling a friend. People necessarily take their bodies for granted as tools for accomplishing some desired purpose; if talking required deliberate musculoskeletal coordination, people would be continually distracted from the message they were trying to convey. The depth of tacit awareness and coordination involved in routine bodily movements is often appreciated only after it breaks down. The patient with Bell's palsy has her life disrupted because essential actions such as speaking and eating are no longer routine. Eating demands constant attention to the possibility of drooling, and her dry right eye is a continually irritating distraction. The extent of the body's taken-for-grantedness in human thought and action is made clear in extreme cases of pain or sickness, during which a patient's deliberate daily effort may be largely consumed with basic functions such as breathing, talking and staying awake.

The directional nature of tacit knowing also highlights the central role of the body in communication. Direct, face-to-face human interaction comprises a rich, highly nuanced exchange of information; the wealth of verbal and non-verbal communication [12] it includes is necessarily absent from written records, telephone interactions and subsequent memories of an interaction. The full range of tacit and explicit information about a patient's particular problem or illness is accessible only from within the clinical encounter. A video recording of an encounter conveys more information than an audio recording, which in turn is more detailed than a written medical record; the information omitted as one moves away from the immediate clinical encounter is often significant [13]. Clinical medicine involves interacting with and understanding persons, and thus addresses a problem that is fundamentally different from and conceptually more complex than the kind of reasoning involved in problems such as mathematical calculation or measuring the masses of chemical isotopes [14].

The pervasiveness of tacit knowing and its taken-for-granted relationship with explicit knowing explains why humans cannot 
use wholly explicit, completely formalizable knowledge. Human knowledge can be conceptualized as a gradual extension from the knower's body to the object or idea of interest in the outside world. This basic structure pertains to all human interactions in the world, from eating breakfast to making sense of facial paralysis.

\section{Tacit knowing in philosophy}

Polanyi turned to philosophy from his career as a physical chemist because he was interested in understanding the process of scientific discovery and problem solving [11]. The dominant understanding of scientific discovery then (and now) was based on Karl Popper's hypothetico-deductive model, in which scientific knowledge comprises information that is potentially falsifiable using empirical data [15]. Polanyi's philosophy began as a refutation of this positivist tradition. He recognized that scientists' ability to identify and solve important problems involves finding a gap or contradiction in an area of knowledge and then working to identify a new, better conception of reality that resolves or explains that gap. Polanyi demonstrated that humans' ability to follow tacit, unspecifiable clues to successful novel solutions accounts for their ability to solve problems and make discoveries [16]. Humans' ability to take the tacit dimension into account fundamentally distinguishes human thinking from the mindless processing of data that machines perform. Polanyi's work has had an important impact on the philosophy of science. Thomas Kuhn's notions of paradigm shifts and scientific progress in The Structure of Scientific Revolutions were heavily influenced by Polanyi's work on problem solving [17]. Polanyi has also influenced other important philosophers of science, such as Paul Feyerabend and Mary Midgley.

Other philosophers have developed ideas that relate to the concept of tacit knowing. The notion of taken-for-grantedness in Alfred Schutz's investigation of human experience [18] is similar to and in some respects more robust than Polanyi's tacit knowing. Schutz, who was concerned with the social structure of knowledge, recognized that in everyday life people explore and solve problems only to the extent necessary for accomplishing the task at hand. People's motivations and existing stock of knowledge determine whether problems are sufficiently relevant to merit attention. Many irrelevant or technical problems are taken for granted and not critically evaluated or even noticed. A patient who works as a fashion model will be extremely motivated to understand and maximize her prognosis for recovery from Bell's palsy, whereas a patient who works in a factory may care little about her appearance but be very anxious to know whether she has a progressive or life-threatening condition. Taken-for-grantedness is even built into the clinical encounter. Patients often assume the clinician's competence and desire to help unless something calls that assumption into question. The experienced doctor may take for granted that the patient's Bell's palsy is typical and so discount inconsistencies in her physical exam as irrelevant to the (already solved) problem of diagnosis [19].

\section{Tacit knowing in psychology and the social sciences}

The concept of tacit knowing is also closely related to current research on reasoning, skill and expertise in medicine. Recent studies of medical skill and expertise have examined clinicians' tendency to form diagnoses rapidly with little or no formal analysis [20,21]. In general novice clinicians rely more heavily on deliberate analytic reasoning, whereas expert clinicians are more likely to use rapid approaches with minimal deliberation or reflection. These snap judgments seem to involve linking new problems with prototypical past examples and become more prevalent with increasing clinical experience [21]. This kind of immediate experiential knowledge has been studied mostly as it relates to visual pattern recognition [22,23], which, like Polanyi's work, draws heavily on insights from Gestalt psychology. These insights from research on skill and expertise generally support Polanyi's argument that skills are acquired primarily tacitly, through practice and apprenticeship, rather than by following explicit rules $[9,24]$.

Clinicians' tendency to make judgments quickly has also received attention in the research on decision making pioneered by Kahneman and Tversky known as 'heuristics and biases' $[25,26]$. This research comprises a large body of evidence demonstrating that people's estimations of probability tend to differ from actual, statistically calculated probabilities in predictable ways. The heuristics and biases programme bears on the common criticism that tacit knowing is an appeal to special knowledge or mystical insight [27]. It is often conflated with that common nostrum 'the art of medicine', which reinforces the mistaken impression that tacit knowing is less rigorous or important than explicit knowing [1]. As shown in the example of skin colour, whether information is tacit or explicit has less to do with its content than it does with how it functions in a particular situation. Tacit knowing is, like all knowledge, neither inerrant nor completely unreliable. The heuristics and biases programme can be interpreted as identifying and explicitly examining common tacit misperceptions in human statistical reasoning. A clinician who learns that he tends to disregard regression to the mean might then explicitly anticipate situations where that tendency will lead him astray so he can avoid incorrect conclusions.

The heuristics and biases programme has demonstrated that humans' tacit knowledge of statistics is unreliable, but cognitive heuristics work quite well for many other kinds of complex judgments [28], such as distinguishing men from women and recognizing other people's emotions [29]. Schutz and Luckmann point out that people tend to take for granted knowledge they have no reason to question or that is irrelevant to the problem at hand [18], so it stands to reason that tacitly appreciated knowledge, while far from perfect, will tend to be more accurate than chance in many situations.

\section{Implications of the tacit dimension for clinical medicine}

As described above, clinicians' ability to incorporate tacit particulars into decisions without employing a deliberate analytic approach is an important, unavoidable source of both practical expertise and systematic error in clinical medicine. Tacit knowing is a part of even seemingly straightforward medical tasks, such as diagnosing Bell's palsy, where subtleties of interpersonal communication and physical examination are often relatively unimportant for treatment decisions. Interpreting diagnostic tests, reviewing the mechanism of Bell's palsy and interpreting data on the efficacy of 
steroids in Bell's palsy all presuppose domains of tacit knowing that are different from but no less necessary than the tacit dimensions of interpersonal communication and clinical experience [10]. Tacit knowing is not a tool or method clinicians can use, nor is it an alternative to explicit medical knowledge. It is the indispensable foundation from which humans make sense of the world [30].

Interpreting and applying explicit information about Bell's palsy requires taking for granted a great deal about the meaning of that information and the conditions under which it is relevant to a particular patient. Formal diagnostic categories, clinical algorithms, and rules of evidence can only pertain 'in general' or 'all else being equal'. That is, only when the conditions necessary for those algorithms and rules to apply are met. Clinicians by definition tend not to notice these mostly tacit assumptions unless they break down. Diagnosing Bell's palsy takes for granted that stroke and trauma have been excluded as diagnoses, and that spots on the patient's chin are acne rather than vesicles from an underlying shingles outbreak. A clinical trial of Bell's palsy treatments always informs treatment decisions, except when it does not because of methodological flaws or results that are not generalizable to certain patients. Decisions about what constitute methodological flaws also have a tacit component, as would methods for clarifying judgments about decisions on those flaws, and so on ad infinitum $[10,31]$.

Reaching a reasonable decision about how to treat a patient's Bell's palsy must also account for the fact that both parties in the clinical encounter are persons. Calls for patient-centred medicine [32] and focus on the clinical encounter [27] are not new, but most such attempts have not questioned the standard view that knowledge comprises only explicit information amenable to formal analysis and so have tended to neglect categories of knowledge with significant tacit components [33]. A clinician's non-verbal communication, for example, affects the patient's emotions and her decision to trust the clinician or not. Non-verbal communication also affects treatment plans. In a thin woman with Bell's palsy the clinician might fail to illicit a family history that suggests risk for steroid-induced diabetes, or he might notice her hesitant speech and discover she is worried about a possible brain tumour. These personal aspects of communication tend to be less amenable to measures and numbers than most physiologic parameters, but they are no less important. The initial case vignette captures very little of what actually goes on between the patient and clinician when they discuss her problem. Other characteristics of personhood are even more difficult to study. All persons have, for instance, relationships with themselves, cultural backgrounds, secret lives, emotions, perceived futures, habits, transcendent dimensions and personal roles that may affect their illness but are nearly impossible to evaluate or even detect with the techniques typically used in medicine and medical research [34]. Developing and analysing comprehensive taxonomies of personal characteristics and how they relate to medicine therefore would require a significant research enterprise involving disciplines far removed from clinical medicine. For clinicians with more modest ambitions, real-time observational research offers one important, underutilized means of figuring out what actually goes on in the clinical encounter because it can potentially identify tacit dimensions of interpersonal communication and examine them explicitly [33].

The complex kinds of information and reasoning involved in the clinical encounter require a frank acknowledgement of uncertainty in medicine. The inescapable tacit component of clinical judgment shows that medical practice is uncertain not only because clinicians and patients never have complete information, but also because they rely on much of the knowledge they do have only tacitly, in ways they cannot fully articulate. Uncertainty is further compounded because the medical knowledge needed to recognize Bell's palsy comes from clinical experience, which allows for faster, more accurate clinical judgment but also introduces additional possibilities for unrecognized errors. Responsible clinical judgment even in this relatively straightforward encounter thus requires not only caution but also humility about the limits of medical knowledge and the certainty of any medical decision. The need for caution is even more important when decisions are reached without firsthand knowledge of the clinical encounter. Accurate judgments about medical decisions are possible using written records or administrative data, but people often fail to appreciate that such records capture only a small portion of the information available in the clinical encounter. Many current measures in the US health care system, for example, are simplistic metrics that cannot distinguish appropriately individualized patient care from care that is wasteful or even harmful [35].

At the start of this paper I suggested that failure to recognize tacit knowing contributes to a gulf between the theory and practice of clinical medicine. How can something so fundamental to all aspects of clinical medicine remain almost completely ignored by clinicians who rely on it every day to care for patients? The answer lies in the structure of tacit knowing. People tend to ignore or take for granted knowledge at the periphery of their attention. A clinician attends carefully to the patient's body, but he is almost totally oblivious to his own body's tacit role in his evaluation of the patient. The body itself tends to be ignored in enterprises ranging from riding a bicycle to philosophical reflection [36]. In fact successfully practicing medicine (as opposed to thinking about how one practices medicine) requires clinicians to concentrate on the patient and take their own bodies for granted. The tacit dimension is so easily ignored that clinicians sometimes have trouble even recognizing it exists, let alone that it has any real or practical importance. Montgomery describes the consequent persistence of the reductionist ideal and belief in completely explicit medical knowledge as an 'epistemological scotoma' that blinds practitioners to the personal and tacit components of their own medical practice [1].

\section{Conclusions}

The same insight that explains why clinicians typically overlook the tacit dimension can predict common criticisms of the argument that practicing clinicians should pay more careful attention to medical epistemology. A busy, pragmatic clinician might argue that if he already takes tacit knowing into account when he correctly diagnoses Bell's palsy, investing additional time to understand how he does so is unnecessary. Others might misinterpret focus on the importance of tacit knowing in the clinical encounter as an attempt to preclude any third party evaluations of medical decisions or restrictions on clinical practice. Finally, many researchers will point to existing studies on decision making that demonstrate how people not only repeatedly make the same kinds of cognitive errors but also have remarkable difficulty explicitly recognizing or correcting them [37,38]. These researchers may 
recognize the function of tacit knowing, but consider it so unreliable that it should be avoided as much as possible.

The latter criticism is particularly relevant to the evidence from the heuristics and biases programme demonstrating that people have poorly developed statistical instincts. It does not necessarily follow, however, that people's numerical estimates are always wrong or that they reason poorly in other domains important to clinical medicine. Past experience with patterns of cranial nerve dysfunction allows clinicians to diagnose Bell's palsy without leafing through medical textbooks; this ability depends on clinicians' ability to take tacit knowing into account. Efforts to avoid the tacit dimension by downplaying clinical expertise merely change the kinds of information that clinicians take for granted $[10,14,31]$. Single-mindedly avoiding tacit errors in Bayesian reasoning will not improve clinical judgment if it comes at the cost of ignoring details of study design that make a clinical trial's results inapplicable to most patients. The typical statistical examples used to illustrate cognitive biases often give the impression that clinical problems have a single correct answer. Problems involving persons are rarely so straightforward because of the range of medical, social and personal factors that patients and clinicians must navigate in the clinical encounter. More often clinicians and patients choose from among several reasonable options after excluding many unreasonable or harmful ones. Foregoing steroid treatment for Bell's palsy may be reasonable in a patient with mild symptoms and poorly controlled diabetes; a magnetic resonance imaging may be reasonable if the patient is unable to sleep or function at work because of anxiety about brain tumours. Finally, different clinicians will have different perspectives about how the dangers of unrecognized bias stack up against the utility of clinical experience. A primary care doctor who has seen several patients recover from Bell's palsy might feel his time-tested approach is effective. A neurologist who sees patients after they fail to improve on acyclovir may be frustrated that clinicians persist in using a treatment for Bell's palsy that has no proven efficacy. A statistician may feel that neither general practitioners nor neurologists have sufficient statistical power to allow meaningful conclusions from their personal clinical experience with Bell's palsy.

Naïve beliefs about medical knowledge can have deleterious practical consequences. The mistaken belief that legitimate medical knowledge comprises only explicitly quantifiable or reliable information is often accompanied by the corollary that information that is mostly tacit is mostly unreliable and so a poor basis for medical decisions. Clinicians who hear this advice often enough may eventually draw the logical conclusion that the tacit dimension should not only be ignored, but that information that is difficult to quantify is likely biased and should be actively suppressed. Clinicians who downplay their judgments about important topics such as non-verbal communication, severity of facial paralysis, trustworthiness, overall health, and patient sophistication because the basis of these judgments are difficult to describe explicitly are throwing away much of the information necessary for making individualized, person-centred decisions. These assumptions about medical knowledge also affect aspects of medicine outside the clinical encounter. They have contributed to the recent epidemiologic turn in medicine whereby discussions of information are often implicitly restricted to quantitative data and treated separately from concepts such as patients' values, preferences, and beliefs [39]. In health policy, focus on information's measurability or concreteness can obscure the distinction between cost effectiveness and cost containment because discussions of these very different goals tend to employ similar quantitative methods [14]. More detailed reporting rules and methodological requirements for published research cannot address the significant tacit influence that industry funding exerts on research design and interpretation of results.

Clinicians who can understand epistemology in medicine will enjoy tangible benefits beyond the intellectual satisfaction of being able to think about medical knowledge in a way that corresponds more closely to the realities of medical practice. Appreciation for the tacit dimension in human knowledge will help clinicians to build a more accurate critical framework for evaluating what kinds of information are important for particular clinical decisions. Recognizing the clinical encounter as an interaction between persons provides better insight into the kinds of largely tacit information that are important for promoting appropriately patient-centred (and person-centred) care. Being able to talk and think explicitly about the function of tacit knowing in clinical judgment will also help clinicians to evaluate more clearly their own medical reasoning and guard against unclear thinking. Clinicians who justify decisions with vague appeals to 'the art of medicine' or 'gut feelings' likely misunderstand the advantages and pitfalls that reliance on the tacit dimension entails. Similarly people who promote one-size-fits all solutions to complex clinical problems are almost certainly ignoring or suppressing the inescapable uncertainty that attends all medical judgment. Recognizing tacit knowing in medicine will make it more difficult for clinicians to convince themselves that knotty problems in medical practice have simple solutions, but it will at least put them in a position to tackle these problems in the light of day rather than in the shadow of misleading epistemological ghosts.

\section{Acknowledgements}

This work was supported by the Robert Wood Johnson Foundation Clinical Scholars Program and the United States Department of Veterans Affairs.

\section{References}

1. Montgomery, K. (2006) How Doctors Think: Clinical Judgment and the Practice of Medicine. New York: Oxford University Press.

2. MacIntyre, A. (1977) Utilitarianism and cost-benefit analysis: an essay on the relevance of moral philosophy to bureaucratic theory. In Values in the Electric Power Industry (ed. K. Sayre), pp. 217-237. Notre Dame: Notre Dame University Press.

3. Pellegrino, E. D. \& Thomasma, D. C. (1981) A Philosophical Basis of Medical Practice. New York: Oxford University Press.

4. Waymack, M. H. (2009) Yearning for certainty and the critique of medicine as 'science'. Theoretical Medicine and Bioethics, 30 (3), 215-229.

5. Klein, P. D. (1998) Epistemology. In Routledge Encyclopedia of Philosophy (ed. E. Craig), pp. 362-365. New York: Routledge.

6. Malterud, K. (1995) The legitimacy of clinical knowledge - towards a medical epistemology embracing the art of medicine. Theoretical Medicine, 16 (2), 183-198.

7. Stephens, G. G. (1988) Reflections of a post-flexnerian physician. In The Task of Medicine: Dialogue at Wickenburg (ed. K. L. White), pp. 172-189. Menlo Park, CA: Henry J. Kaiser Family Foundation. 
8. Sturmberg, J. P. \& Martin, C. M. (2008) Knowing - in medicine. Journal of Evaluation in Clinical Practice, 14 (5), 767-770.

9. Polanyi, M. (1962) Personal Knowledge: Towards a Post-Critical Philosophy. London: Routledge and Kegan Paul.

10. Thornton, T. (2006) Tacit knowledge as the unifying factor in evidence based medicine and clinical judgment. Philosophy, Ethics, and Humanities in Medicine, 1 (1), E2.

11. Polanyi, M. (1966) The Tacit Dimension. Garden City, NY: Doubleday \& Company.

12. Knapp, M. L. \& Hall, J. A. (2009) Nonverbal Communication in Human Interaction. Boston, MA: Wadsworth, Cengage Learning.

13. Riddle, D. L., Albrecht, T. L., Coovert, M. D., Penner, L. A., Ruckdeschel, J. C., Blanchard, C. G., Quinn, G. \& Urbizu, D. (2002) Differences in audiotaped versus videotaped physician-patient interactions. Journal of Nonverbal Behavior, 26, 219-240.

14. Henry, S. G., Zaner, R. M. \& Dittus, R. S. (2007) Viewpoint: moving beyond evidence-based medicine. Academic Medicine, 82 (3), 292 297.

15. Popper, K. R. (1959) The Logic of Scientific Discovery. New York: Basic Books.

16. Polanyi, M. (1997) Creative imagination. In Michael Polanyi, Society, Economics, and Philosophy: Selected Papers (ed. R. T. Allen), pp. 249-266. New Brunswick, NJ: Transaction Publishers.

17. Moleski, M. X. (2007) Polanyi vs. Kuhn: worldviews apart. Tradition and Discovery, 33 (2), 8-24.

18. Schutz, A. \& Luckmann, T. (1973) The Structures of the Life-world. Evanston, IL: Northwestern University Press.

19. Schwartz, M. A. \& Wiggins, O. P. (1988) Scientific and humanistic medicine: a theory of clinical methods. In The Task of Medicine: Dialogue at Wickenburg (ed. K. L. White), pp. 137-171. Menlo Park, CA: The Henry J. Kaiser Family Foundation.

20. Norman, G., Young, M. \& Brooks, L. (2007) Non-analytical models of clinical reasoning: the role of experience. Medical Education, 41 (12), $1140-1145$.

21. Norman, G., Eva, K., Brooks, L. \& Hamstra, S. (2006) Expertise in medicine and surgery. In The Cambridge Handbook of Expertise and Expert Performance (eds. K. A. Ericsson, N. Charness, P. Feltovich \& R. Hoffman), pp. 339-353. Cambridge; New York: Cambridge University Press.

22. Bleakley, A., Farrow, R., Gould, D. \& Marshall, R. (2003) Making sense of clinical reasoning: judgment and the evidence of the senses. Medical Education, 37 (6), 544-552.

23. Engel, P. J. H. (2008) Tacit knowledge and visual expertise in medical diagnostic reasoning: implications for medical education. Medical Teacher, 30, e184-e188.
24. Klein, E. P. (2002) Toward a Skills-based Philosophy of Medicine. [Ph.D. Dissertation]. Washington, DC: Georgetown University.

25. Kahneman, D., Slovic, P. \& Tversky, A. (1982) Judgment under Uncertainty: Heuristics and Biases. Cambridge: Cambridge University Press.

26. Gilovich, T., Griffin, D. W. \& Kahneman, D. (2002) Heuristics and Biases: The Psychology of Intuitive Judgment. New York: Cambridge University Press.

27. Pellegrino, E. D. (1979) The anatomy of clinical judgments: some notes on right reason and right action. In Clinical Judgment: A Critical Appraisal (eds H. T. Engelhardt Jr, S. F. Spicker \& B. Towers), pp. 169-194. Boston, MA: D. Reidel Publishing Company.

28. Gigerenzer, G., Czerlinkski, J. \& Martignon, L. (2002) How good are fast and frugal heuristics? In Heuristics and Biases: The Psychology of Intuitive Judgment (eds T. Gilovich, D. Griffin \& D. Kahneman), pp. 559-581. New York: Cambridge University Press.

29. Ekman, P. \& Friesen, W. V. (2003) Unmasking the Face: A Guide to Recognizing Emotions from Facial Clues. Cambridge, MA: Malor Books.

30. Grene, M. (1977) Tacit knowing: grounds for a revolution in philosophy. Journal of the British Society for Phenomenology, 8, 164-171.

31. Braude, H. D. (2009) Clinical intuition versus statistics: different modes of tacit knowledge in clinical epidemiology and evidence-based medicine. Theoretical Medicine and Bioethics, 30 (3), 181-198.

32. Stewart, M., Brown, J. B., Weston, W. W., McWhinney, I. R., McWilliam, C. L. \& Freeman, T. R. (1995) Patient-Centered Medicine: Transforming the Clinical Method. Thousand Oaks, CA: Sage Publications.

33. Henry, S. G. (2006) Recognizing tacit knowledge in medical epistemology. Theoretical Medicine and Bioethics, 27 (3), 187-213.

34. Cassell, E. J. (2004) The Nature of Suffering and the Goals of Medicine. Oxford; New York: Oxford University Press.

35. Hayward, R. A. (2008) Access to clinically-detailed patient information: a fundamental element for improving the efficiency and quality of healthcare. Medical Care, 46 (3), 229-231.

36. Leder, D. (1990) The Absent Body. Chicago, IL: University of Chicago Press.

37. Pronin, E. (2007) Perception and misperception of bias in human judgment. Trends in Cognitive Science, 11 (1), 37-43.

38. Einhorn, H. J. \& Hogarth, R. M. (1978) Confidence in judgment persistence of illusion of validity. Psychological Review, 85 (5), 395 416.

39. Straus, S. E., Richardson, W. S., Glasziou, P. \& Haynes, R. B. (2005) Evidence-Based Medicine: How to Practice and Teach EBM, 3rd edn. Philadelphia, PA: Elsevier Churchill Livingstone. 